\title{
Systematic behaviours of different quantities related to sequential prompt emission in fission
}

\author{
Anabella Tudora, ${ }^{1, *}$ \\ ${ }^{1}$ University of Bucharest, Faculty of Physics, 405 Atomistilor Str., POB MG-11, RO-77125, Bucharest-Magurele, Romania
}

\begin{abstract}
The deterministic modelling of sequential prompt emission in fission based on recursive equations of residual temperature was applied to numerous fission cases. This fact emphasized systematics and correlations between different quantities characterizing the residual fragments and the sequential emission. General forms of residual temperature distributions for each emission sequence are determined on the basis of these systematics, having as application the inclusion of sequential emission into the Los Alamos model. Also the systematics can serve to obtain indicative values of different average quantities in the absence of any prompt emission model.
\end{abstract}

\section{Brief mentions about the modelling}

A deterministic modelling of sequential prompt emission in fission was recently developed (see Ref.[1] for details). This treatment is based on recursive transcendent equations of the nuclear temperature of residual fragments. By solving such equations for each emission sequence " $k$ " corresponding to each initial (pre-neutron) fragment $\{\mathrm{A}, \mathrm{Z}\}$ at each TKE value of the fragmentation and TKE ranges, multi-parametric matrices of different quantities -generically labelled $\mathrm{q}_{\mathrm{k}}(\mathrm{A}, \mathrm{Z}, \mathrm{TKE})$ - characterizing the initial and residual fragments and the prompt emission are obtained (e.g. nuclear temperature $\mathrm{T}_{\mathrm{k}}(\mathrm{A}, \mathrm{Z}, \mathrm{TKE})$ and excitation energy $\mathrm{E}_{\mathrm{k}}(\mathrm{A}, \mathrm{Z}, \mathrm{TKE})$, average prompt neutron energy in the centre-of-mass frame $\langle\varepsilon\rangle_{\mathrm{k}}(\mathrm{A}, \mathrm{Z}, \mathrm{TKE})$ etc.). These quantities appear with the probability expressed by the fragment distribution Y(A,Z,TKE).

The sequential emission modelling was applied to a large number of fission cases for which reliable experimental data of $\mathrm{Y}(\mathrm{A}, \mathrm{TKE})$ distributions exist. The 49 studied cases include the spontaneous fission of ${ }^{252} \mathrm{Cf}$ and ${ }^{236,238,240,242,244} \mathrm{Pu}$, the thermal neutron induced fission of ${ }^{233,235} \mathrm{U}$ and ${ }^{239} \mathrm{Pu}$ and the fast neutron induced fission of ${ }^{234,238} \mathrm{U}$ and ${ }^{237} \mathrm{~Np}$ at incident energies below the threshold of the second chance fission.

For each fissioning nucleus the initial fragmentation range was deterministically constructed as in the Pointby-Point $(\mathrm{PbP})$ treatment (see Ref.[2] and references therein). I.e. a large fragment mass range was considered (with A going from symmetric fission up to a very asymmetric split). Five charge numbers $\mathrm{Z}$ were taken for each $A$ as the nearest integer values above and below the most probable charge $\mathrm{Zp}(\mathrm{A})=\mathrm{Z}_{\mathrm{UCD}}(\mathrm{A})+\Delta \mathrm{Z}(\mathrm{A})$. A large TKE range was taken for each fragmentation, e.g. TKE from 100 to $200 \mathrm{MeV}$ with a step size of $5 \mathrm{MeV}$.
The same method of total excitation energy (TXE) partition based on modelling at scission which is used in the $\mathrm{PbP}$ treatment (Ref.[2] and references therein) is employed in this model, too.

Different prescriptions concerning the compound nucleus cross-section of the inverse process of neutron evaporation $\sigma_{\mathrm{c}}(\varepsilon)$ and the level density parameter of initial and residual fragments were used.

\section{Systematic behaviours}

The large number of studied cases has emphasized systematics and correlations between different average quantities, briefly mentioned in the following.

It was ascertained that for each emission sequence the ratios of the average residual to initial temperature $\left\langle\mathrm{T}_{\mathrm{k}}\right\rangle\left|<\mathrm{T}_{\mathrm{i}}\right\rangle$ is almost the same for all studied cases without depending on the prescriptions used for compound nucleus cross-section of the inverse process $\sigma_{c}(\varepsilon)$ and the level density parameters. The upper part of Fig.1 shows the ratios $\left\langle\mathrm{T}_{\mathrm{k}}\right\rangle \mid\left\langle\mathrm{T}_{\mathrm{i}}\right\rangle$ as a function of $<$ TXE $>$ plotted with different coloured symbols corresponding to the emission sequences with $\mathrm{k}$ from 1 to 5 and to all sequences (black stars). The constant values approximating these ratios are represented by horizontal lines plotted with the same colour as the respective symbol. The ratios of the average residual energy to the initial excitation energy $\left\langle\mathrm{E}_{\mathrm{k}}>/ \mathrm{E}^{*}\right\rangle$ exhibit a similar behaviour as it can be seen in the lower part of Fig.1. In both cases the constant values of the ratios for $\mathrm{k}=1$ and 2 corresponding to the light and heavy fragment groups are equal. The values of the residual temperature and energy ratios of all emission sequences are just the mean of the constant values for the sequences $\mathrm{k}=1$ and 2 , because the first two emission sequences take place for the majority of initial fragments at a great part of the TKE values. For $k>2$ the constant values of the 
temperature and energy ratios are higher for light fragments than for heavy ones, because of a larger difference between the residual energies of light and heavy fragments $\Delta \mathrm{E}_{\mathrm{k}}=\left\langle\mathrm{E}_{\mathrm{k}}\right\rangle_{\mathrm{L}}-\left\langle\mathrm{E}_{\mathrm{k}}\right\rangle_{\mathrm{H}}$ (which is of about $1.5 \mathrm{MeV})$ compared to the first two sequences $(\mathrm{k}=1,2)$ for which this difference is less than $1 \mathrm{MeV}$.
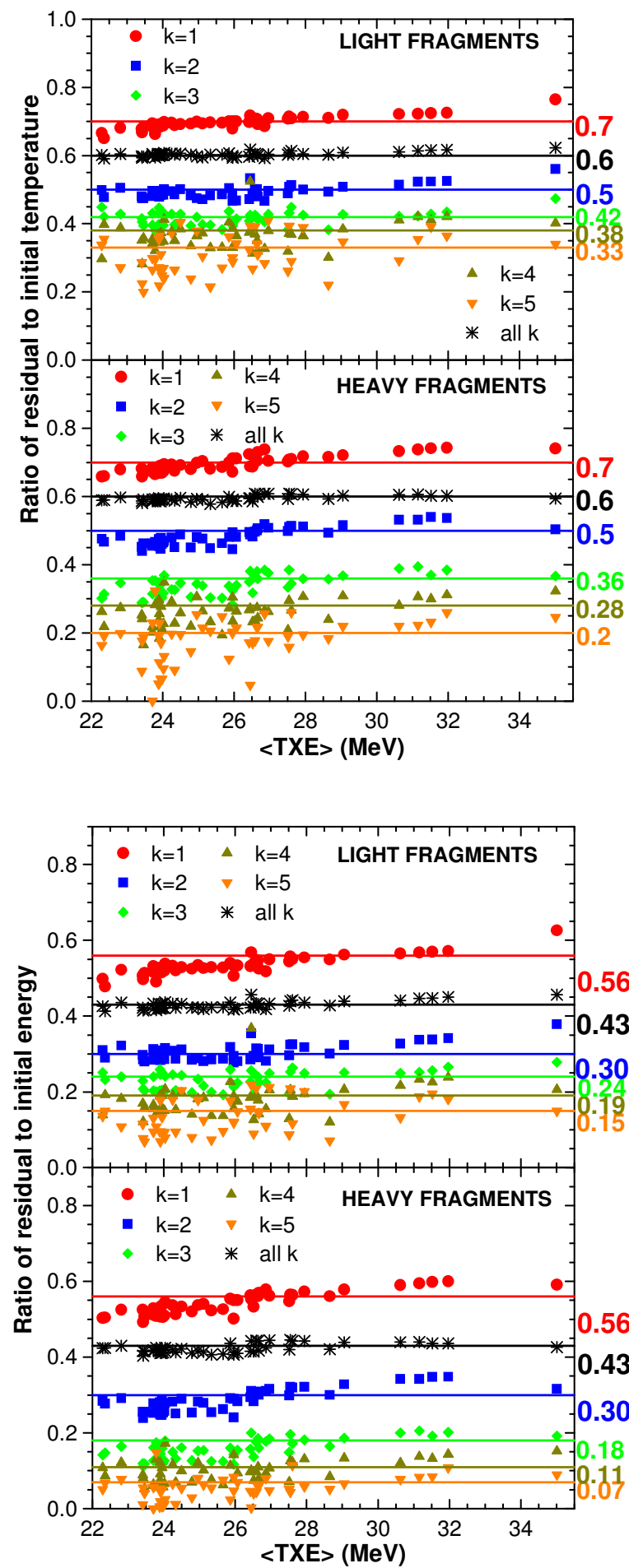

Fig.1: The ratios of residual temperature (upper part) and residual energy (lower part) to those of initial fragments corresponding to each sequence $\mathrm{k}$ (different coloured symbols) and to all emission sequences (black stars) and the constant values approximating these ratios (horizontal lines plotted with the same colour as the respective symbol).
Linear correlations between the average centre-ofmass energy of each emitted neutron $\langle\varepsilon\rangle_{k}$ and the corresponding average residual temperature $\left\langle\mathrm{T}_{\mathrm{k}}\right\rangle$ (see Fig.2) and between $\langle\varepsilon\rangle_{k}$ and the square-root of $\left\langle E_{k}\right\rangle$ are observed. The slopes of these linear dependences allow the determination of global values for the total average level density parameter of the light and heavy fragment groups. E.g. the obtained global values $<\mathrm{a}>_{\mathrm{L}}=12 \mathrm{MeV}^{-1}$ and $\langle\mathrm{a}\rangle_{\mathrm{H}}=11.37 \mathrm{MeV}^{-1}$ are in good agreement with the values resulting from sequential emission calculations (under the prescription of level density parameters provided by the Egidy-Bucurescu systematic for the back shift Fermi-gas model.

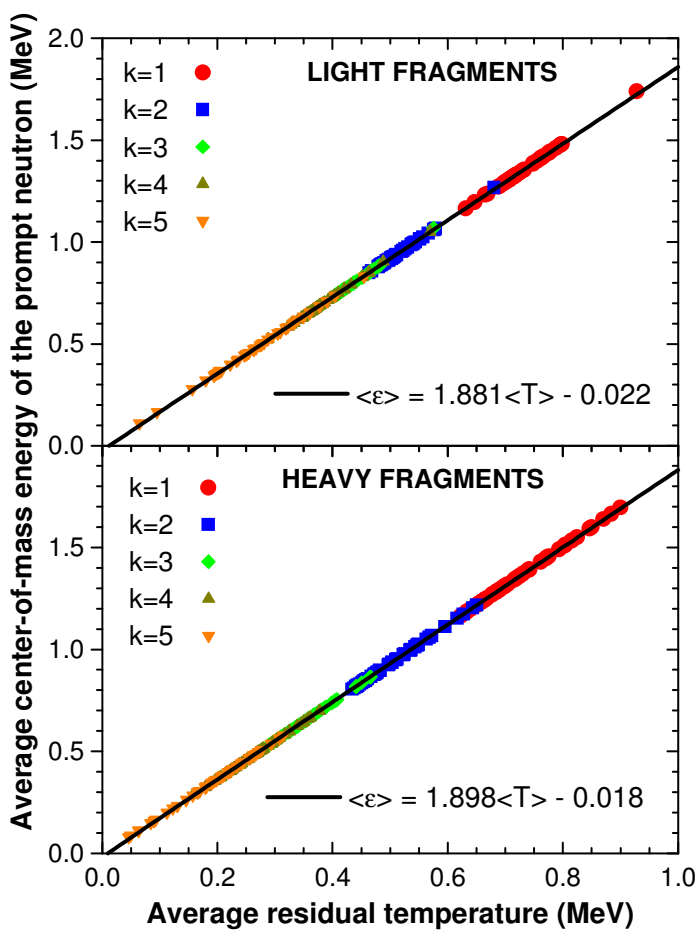

Fig.2: Average centre-of-mass energy of each emitted neutron $\langle\varepsilon\rangle_{\mathrm{k}}$ (different symbols for $\mathrm{k}$ from 1 to 5 ) as a function of the average residual temperature $\left\langle\mathrm{T}_{k}\right\rangle$. The linear fits are given with black lines.

Linear dependences of the average energy carried away per each emitted neutron $\left(\left\langle\eta_{\mathrm{k}}\right\rangle=\langle\varepsilon\rangle_{\mathrm{k}}+\langle\mathrm{Sn}\rangle_{\mathrm{k}-1}\right)$ on the average neutron separation energy from the precursor fragment and of the average energy carried away per neutron corresponding to all emission sequences $\langle\eta\rangle$ on the total average residual temperature $\langle\mathrm{T}\rangle$ and level density parameter $<$ a $>$ are obtained, too.

The systematics mentioned above can be used to obtain indicative values of different prompt emission quantities in the absence of any prompt emission model. For instance if the average temperatures of initial fragments are known then the average prompt neutron energy in the centre-of-mass frame can be obtained from the linear fit of Fig. 2 using the values of the temperature ratios (indicated with horizontal lines) from the systematic given in Fig.1. Two examples for ${ }^{252} \mathrm{Cf}(\mathrm{SF})$ for which 
$<\mathrm{TXE}\rangle=35.01 \mathrm{MeV},\left\langle\mathrm{E}^{*}\right\rangle_{\mathrm{L}}=19.97 \mathrm{MeV}$ and $\left\langle\mathrm{E}^{*}\right\rangle_{\mathrm{H}}=$ $15.04 \mathrm{MeV}$ are given: a) using the equivalent initial temperature in which the average level density parameter is approximated by $\langle\mathrm{a}\rangle=\mathrm{A}_{0} / 11 \mathrm{MeV}^{-1}$, the value $\langle\varepsilon\rangle=$ $1.38 \mathrm{MeV}$ is obtained, in agreement with the experimental data; b) considering the average initial temperatures $\left\langle\mathrm{T}_{\mathrm{i}}\right\rangle_{\mathrm{L}, \mathrm{H}}$ based on the average level density parameters given by the super-fluid model $\left\langle\mathrm{a}_{\mathrm{L}}\right\rangle=13.55$ $\mathrm{MeV}^{-1}$ and $\left\langle\mathrm{a}_{\mathrm{H}}\right\rangle=12.76 \mathrm{MeV}^{-1}$, the values $\langle\varepsilon\rangle_{\mathrm{L}}=1.43$ $\mathrm{MeV},\langle\varepsilon\rangle_{\mathrm{H}}=1.27 \mathrm{MeV}$ and $\langle\varepsilon\rangle=1.36 \mathrm{MeV}$ are obtained, also in reasonable agreement with the experimental data and model calculations.

\section{Inclusion of sequential emission into the Los Alamos model}

The most important feature of the Los Alamos (LA) model, i.e. the most probable fragmentation approach, consists of the global treatment of sequential emission using a residual temperature distribution $\mathrm{P}(\mathrm{T})$ on which the centre-of-mass energy spectrum at a given residual temperature is integrated. Up to now the maximum temperature $\left(\mathrm{T}_{\max }\right)$ of the triangular form of $\mathrm{P}(\mathrm{T})$ was taken equal to the nuclear temperature of initial fragments [2] - [4], either an equivalent initial temperature of both fragments as in the LA model of Madland and Nix [3] (including the subsequent improvements):

$$
T_{\max }=<T_{i}>_{\text {equiv }}=\sqrt{<T X E>/<a>}
$$

or different initial temperatures of complementary fragments as in the LA model of Madland and Kahler [4]:

$$
T_{\max }=\left\langle T_{i}\right\rangle_{L . H}=\sqrt{\left\langle E_{L . H}^{*}>/<a_{L, H}>\right.}
$$

In the PbP model (Ref.[2] and references therein) $\mathrm{T}_{\max }$ is taken as the temperature of each initial fragment of the fragmentation range at each TKE value:

$$
T_{i}(A, Z, T K E)=\sqrt{E *(A, Z, T K E) / a(A, Z, T K E)}
$$

Taking into account the systematic of the residual temperature ratio corresponding to all emission sequences $\langle\mathrm{T}\rangle \mid\left\langle\mathrm{T}_{\mathrm{i}}\right\rangle=0.6$ (black stars in Fig.1), a triangular $\mathrm{P}(\mathrm{T})$ with $\mathrm{T}_{\max }$ related to the initial temperature $\left(\mathrm{T}_{\max }=0.9<\mathrm{Ti}>\right)$ was proposed in Ref.[1] and it was successfully used in the PbP model, too (see Ref.[1] for details).

The constant values of the residual temperature ratios corresponding to each emission sequence given in Fig.1, i.e. $\left.\left\langle\mathrm{T}_{\mathrm{k}}\right\rangle /<\mathrm{T}_{\mathrm{i}}\right\rangle=\mathrm{r}_{\mathrm{k}}$, allow to define a residual temperature distribution for each emission sequence $\mathrm{P}_{\mathrm{k}}(\mathrm{T})$ with the maximum temperature related only to the temperature of initial fragments by the relation:

$$
T_{\max L, H}^{(k)}=(3 / 2) r_{k}<T_{i}>_{L, H}
$$

in which $r_{k}$ are the constant values indicated by horizontal lines in Fig.1.

Consequently the LA model can be improved again, this time by the inclusion of sequential emission. The centre-of-mass energy spectrum of each neutron successively emitted from the light or heavy fragment is given by the following equation in which the index $\mathrm{L}, \mathrm{H}$ is omitted:

$$
\Phi_{k}(\mathcal{E})=\varepsilon \sigma_{c}(\varepsilon) \int_{0}^{T_{\text {max }}^{(k)}} K_{k}(T) P_{k}(T) \exp (-\varepsilon / T) d T
$$

where the upper limit of the integral $\mathrm{T}_{\max }{ }^{(\mathrm{k})}$ is given by Eq.(4) and the normalization constant is

$$
K_{k}(T)=\left(\int_{0}^{\infty} \varepsilon \sigma_{c}(\varepsilon) \exp (-\varepsilon / T) d \varepsilon\right)^{-1}
$$

The prompt neutron spectrum in the laboratory frame (PFNS) of the k-th prompt neutron (isotropically emitted in the centre-of-mass frame of the mother fragment) is given by the following equation in which the index $\mathrm{L}, \mathrm{H}$ is omitted, too:

$$
N_{k}(E)=\int_{\left(\sqrt{E}-\sqrt{E_{f}}\right)^{2}}^{\left(\sqrt{E}+\sqrt{E_{f}}\right)^{2}} \frac{\Phi_{k}(\varepsilon) d \varepsilon}{4 \sqrt{E_{f} \varepsilon}}
$$

where $\Phi_{\mathrm{k}}(\varepsilon)$ is given by Eq.(5) and $\mathrm{E}_{\mathrm{f}}$ is the kinetic energy per nucleon (the same for all sequences).

The LA model with sequential emission requires as input parameters only the average temperatures of initial fragments (based on the average excitation energies and level density parameters) and the average $<$ TKE $>$. This model can be also used with different prescriptions concerning $\sigma_{\mathrm{c}}(\varepsilon)$ (e.g. provided by optical model calculations, analytical expressions etc.), the TXE partition giving $\left\langle\mathrm{E}^{*}\right\rangle_{\mathrm{L}, \mathrm{H}}$ (different methods), the level density parameters of initial and residual fragments (e.g. non-energy dependent given by systematics, or energydependent provided by the super-fluid model etc.).

Prompt neutron spectrum results of the LA model with sequential emission are illustrated for ${ }^{235} U\left(n_{t h}, f\right)$ taken as an example. In this calculation the following prescriptions were used: $\sigma_{\mathrm{c}}(\varepsilon)$ from optical model calculations with the parameterisation of BecchettiGreenlees, the TXE partition based on modelling at scission usually employed in the PbP model [2] and level density parameters provided by the super-fluid model.

Fig.3 shows the centre-of-mass energy spectrum $\Phi_{\mathrm{k}}(\varepsilon)$ of the first three neutrons (plotted with different dashed and dotted lines) successively emitted from the light and heavy fragment (upper and lower part, respectively) calculated according to Eq.(5), as well as the total centre-of-mass energy spectrum (solid red line) which describes very well the experimental data recently measured by Göök et al. [5].

The total PFNS in the laboratory frame (thick solid red line) together with the contributions of the first (dashed lines), second (dash-dotted lines) and third neutron (dotted lines) emitted from the light fragment (blue colour) and heavy fragment (green colour) and the contribution of all emitted neutrons (thin solid lines) are plotted in Fig.4. For a better visualization, the high and low energy parts of the spectrum are focused in separate frames. 


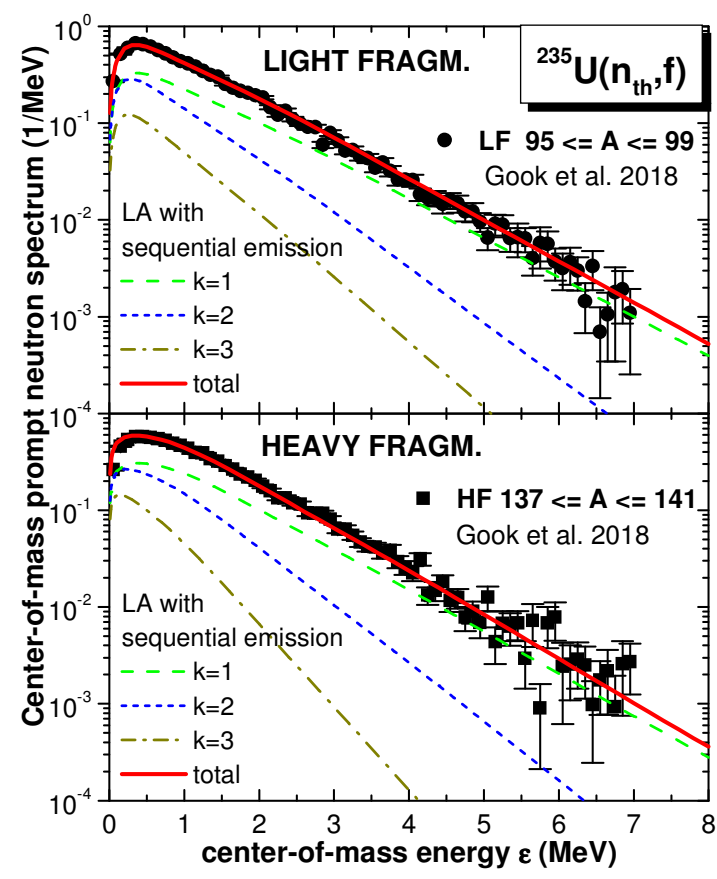

Fig.3: Prompt neutron spectra in the centre-of-mass frame corresponding to the light and heavy fragment group (upper and lower part, respectively): The result of the LA model with sequential emission: $\Phi_{k}(\varepsilon)$ of the first three emitted neutrons (different dashed and dotted lines) and the total $\Phi(\varepsilon)$ (red solid line) describing very well the experimental data of Göök et al.[5] (symbols).

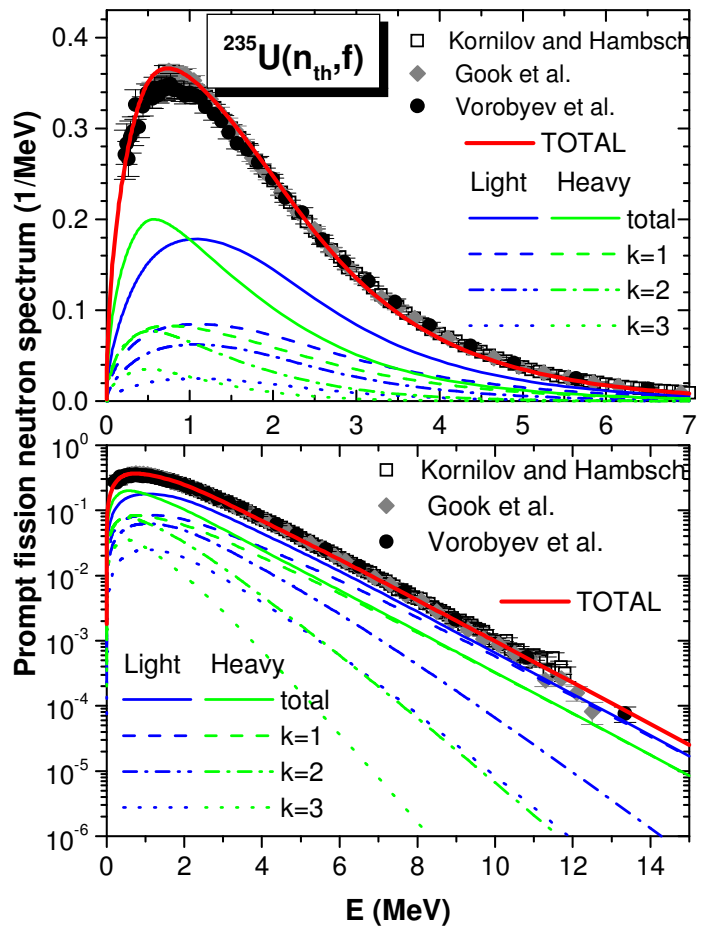

Fig.4: PFNS result of the LA model with sequential emission: spectra of each emitted neutron (different dashed or dotted lines) and of all emitted neutrons (thin solid lines) from the light (blue colour) and heavy (green colour) fragments and the total spectrum (thick solid red line) describing well the experimental data (different symbols).
As it can be seen the total PFNS is obtained in good agreement with the experimental data (different symbols) measured in the last decade.

\section{Conclusions}

The deterministic modelling of sequential emission applied to 49 fission cases allowed to obtain systematics and correlations between different average quantities characterizing the initial and residual fragments and the prompt emission.

1. The residual temperature and energy ratios corresponding to each emission sequence $\left(<\mathrm{T}_{\mathrm{k}}>\left|<\mathrm{T}_{\mathrm{i}}\right\rangle\right.$, $\left.\left\langle\mathrm{E}_{\mathrm{k}}\right\rangle \mid\left\langle\mathrm{E}^{*}\right\rangle\right)$ and to all emission sequences $\left(\langle\mathrm{T}\rangle \mid\left\langle\mathrm{T}_{\mathrm{i}}\right\rangle\right.$, $<\mathrm{E}\rangle \mid\left\langle\mathrm{E}^{*}\right\rangle$ ) are almost the same (constant values) for all investigated fission cases, without depending on the prescriptions used for $\sigma_{\mathrm{c}}(\varepsilon)$ and the level density parameters of the initial and residual fragments.

2. The constant values of the temperature ratios $\left\langle\mathrm{T}_{\mathrm{k}}>/<\mathrm{T}_{\mathrm{i}}\right\rangle=\mathrm{r}_{\mathrm{k}}$ (e.g. $\mathrm{r}_{1}=0.7$ and $\mathrm{r}_{2}=0.5$ for both light and heavy fragment groups, $r_{3}=0.42$ for light fragments and $r_{3}=0.36$ for heavy fragments, etc.) allow to define a residual temperature distribution for each emission sequence $\mathrm{P}_{k}(\mathrm{~T})$ with the maximum temperature related only to the initial temperature $\mathrm{T}_{\max }{ }^{(\mathrm{k})}=(3 / 2) \mathrm{r}_{\mathrm{k}}<\mathrm{T}_{\mathrm{i}}>$, having as application the inclusion of sequential emission into the Los Alamos model.

3. The constant values of the ratios $\left\langle\mathrm{T}_{\mathrm{k}}\right\rangle \mid\left\langle\mathrm{T}_{\mathrm{i}}\right\rangle$ and the linear behaviour of the average centre-of-mass energy of prompt neutrons $\langle\varepsilon\rangle_{\mathrm{L}, \mathrm{H}}$ as a function of $\langle\mathrm{T}\rangle$ allow to obtain indicative values of different prompt emission quantities in the absence of any prompt emission model.

4. The linear dependences of $\langle\varepsilon\rangle_{k}$ on $\left\langle T_{k}\right\rangle$ and of $\langle\varepsilon\rangle_{k}$ on $\left\langle\mathrm{E}_{\mathrm{k}}\right\rangle^{1 / 2}$ are the same for all emission sequences. Almost linear dependences of the energy carried away by each emitted neutron $\left\langle\eta_{k}\right\rangle$ on the neutron separation energy from the precursor $\langle\mathrm{Sn}\rangle_{\mathrm{k}-1}$, on the residual temperature $\left\langle\mathrm{T}_{\mathrm{k}}\right\rangle$ and on the average level density parameter of each residual fragment group are established, too.

Acknowledgements: the present work was done in the frame of the project PN-III-P4 ID-PCE-2016-0014 (contract 7/2017).

\section{References}

1. A. Tudora, F.-J. Hambsch, V. Tobosaru, Eur. Phys. J. A 54, 87 (2018)

2. A. Tudora, F.-J. Hambsch, Eur. Phys. J. A 53, 159 (2017)

3. D.G. Madland, J.R. Nix, Nucl. Sci. Eng. 81, 213 (1982)

4. D.G. Madland, A.C. Kahler, Nucl. Phys. A 957, 289 (2017)

5. A. Göök, F.-J. Hambsch, S. Oberstedt, M. Vidali, Phys. Rev. C 98, 044615 (2018) 